\title{
Socio-Environmental Co-Factors Associated with Cervical Cancer in Bangladesh
}

\author{
Md. Monirul Hassan ${ }^{1 *}$, Dr. Taslima Nigar ${ }^{2}$ \\ ${ }^{1}$ Student, Department of Environmental Science, Jahangirnagar University (JU), Savar, Bangladesh \\ ${ }^{2}$ Junior Consultant, Department of Gynaecological oncology, National Institute of Cancer Research and Hospital, Mohakhali, Dhaka, \\ Bangladesh
}

DOI: $10.36348 /$ sijog.2021.v04i03.003

| Received: 23.02.2021 | Accepted: 05.03.2021 | Published: 21.03.2021

*Corresponding author: Md. Monirul Hassan

\section{Abstract}

Introduction: Cervical cancer is one of the major NCDs around the world and is the second leading malignancy in terms of mortality and incident cases in Bangladesh. The high mortality rate was associated with a lack of awareness regarding cervical cancer. The study was conducted to find out the relations between the incidence levels of cervical cancer, and social and environmental co-factors. The aim of the study was to figure out the socio-environmental factors of cervical cancer patients in Bangladesh and measure their effects on the incidence numbers. Methods: This was a cross-sectional study conducted over 4 months with a sample size of 100. The study was conducted with only female subjects in Dhaka city, among the patients coming to the Genealogical Department of the NICRH. Result: The majority of the patients were above the age of 40 and only $20 \%$ of the patients were under the age of $40.69 \%$ of cancer patients had no education. $31 \%$ had some level of education. $88 \%$ of the cancer patients were housewives. $2 \%$ were involved in agriculture and $10 \%$ were in other occupations. Over half had a monthly income of less than 10,000 BDT. Almost 3/4 ${ }^{\text {th }}$ of the patients got married before the age of 17 . The study subjects had a higher-than-average number of children, with $57 \%$ of them having 3-5 children, $27 \%$ of patients had less than 3 children and $16 \%$ of them had more than 5 children. The majority of the patients were admitted with advanced stages of cancer. Only $2 \%$ had been admitted with stage I disease. Conclusion: The study showed a surprising lack of knowledge of cervical cancer and the socio-environmental factors that play a part in it. Awareness was positively related to education level.

Keywords: Cancer, Cervical Cancer, Demographic, HPV, Screening, Cancer screening, socio-environmental, stage, AFSI.

Copyright (C) 2021 The Author(s): This is an open-access article distributed under the terms of the Creative Commons Attribution 4.0 International License (CC BY-NC 4.0) which permits unrestricted use, distribution, and reproduction in any medium for non-commercial use provided the original author and source are credited.

\section{INTRODUCTION}

Bangladesh is one of the densely populated places in the world. Poverty in Bangladesh has declined remarkably since the early-2000s, and as a result of accelerated economic growth, many new diseases are emerging along with lifestyle changes. According to recent studies, the incidence of cancer has been increasing steeply, especially in countries with lower and middle incomes [1, 2]. In Bangladesh, Cervical cancer is one of the second leading malignancies in terms of both incident cases and deaths. These kinds of high mortality rates have been attributed to our socioenvironmental situation. Many factors from our social demographic, like poverty, lack of awareness, early age of marriage, lack of proper education can be linked to the high number of cancer incidence and mortality rates. Cases of cervical cancer have been found to generally increase along with age and a prolonged period of Sexual Intercourse [3]. Cervical cancer has shown a greater incidence and mortality rate in countries with lower income when compared to other countries. It is often referred to as the disease of the poor, uneducated, and the underserved. This is mostly because women coming from poor financial and educational backgrounds often have less awareness regarding their wellbeing and are less likely to go for routine check-ups and screening [9, 10]. Many studies conducted in similar demographic areas have found a link between employment status and general wellbeing. This is because a stable workplace gives us a sense of accomplishment. Most studies show a relationship 
between the age at first sexual intercourse with a high likelihood of cancer. Environmental co-factors can depend on the economic condition of different countries. In developing or underdeveloped countries, early age marriage and childbirth, poor economic condition, poor hygiene, passive smoking, and oral contraceptive are more prominent factors for cervical cancer. On the other hand, in developed countries, the main factors related to cervical cancer are multiple sexual partners, active smoking, and HIV/AIDS. This study was conducted to find out the specific factors that play a role in cervical cancer, specifically in our country.

\section{OBJECTIVE}

To evaluate the Socio-Environmental Cofactors on cervical cancer patients in Bangladesh

\section{METHODOLOGY AND MATERIALS}

This was a cross-sectional study performed in the gynecological department of NICRH, Bangladesh. A total $\mathrm{f} 100$ cancer patients were selected as sample size after confirmed diagnosis by biopsy of cervical tissue. The study was conducted over a 4-month period, using questionnaires as the data source, and the patients were selected purposively. The patients were made aware of the study and proper consent was taken. A questionnaire was coded before entering the data into the computer by the researcher. The sample was selected purposively to interview the study population considering the inclusion and exclusion criteria. The sample size was selected purposively. The statistical analysis of the data was carried out by using the software program SPSS. Data were being checked, edited, and appropriately coded before analysis. For summarizing data, statistics such as mean, median, and mode, and the percentage were calculated. The data were presented in tables, graphs, and charts. The questionnaires included information on sociodemographic characteristics, smoking history, reproductive history, menstrual factors, and sexual behavior.

\section{Inclusion Criteria}

- Only the female population

- Patients who had given consent to participate in the study.

- Those already diagnosed with cancer

\section{Exclusion Criteria}

- Mentally ill.

- Unable to answer the criteria question.

- Exclude those affected with other chronic diseases etc.

\section{RESULTS}

The complete study was conducted with 100 females only sample size. The majority of the patients were above the age of 40 , and over half the study population belonged to the age group of 41-50. Only $20 \%$ of the patients were under the age of 40 . In this study, the illiteracy rates were extremely high, with $69 \%$ of cancer patients having no education. $27 \%$ had education above primary level and only $4 \%$ had education above the SSC level. $88 \%$ of the cancer patients were simply housewives. $2 \%$ were involved in agriculture and $10 \%$ were in other occupations. A monthly income level of less than 10000 BDT was found in over half the study population. $31 \%$ had a monthly income of less than 25000 , and $15 \%$ cases had an income level of above 25000 BDT. The majority of the patients married early, with $73 \%$ getting married at the early age of $14-17$ years, $19 \%$ in the age group of $18-21$ years, and $8 \%$ in the $22-25$ years group. The study subjects had a higher-than-average number of children, with $57 \%$ of them having 3-5 children, $27 \%$ of patients had 2 or fewer children and $16 \%$ of them had more than 5 children. The majority of the patients were admitted with advanced stages (stage II \& III) of cancer, as $61 \%$ were in stage II and $37 \%$ were in stage III. Only $2 \%$ had been admitted with stage I disease.

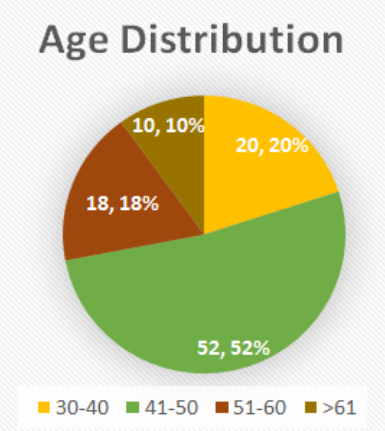

Fig-1: Age distribution of the cervical cancer patients

In this study, most (52\%) of the cancer patients were from the age group of 41-50 years. $20 \%$ of the patients are in the range of $30-40$ years and $16 \%$ in the 51-60 years age group. Only $10 \%$ of the patients are above the age of 61 years.

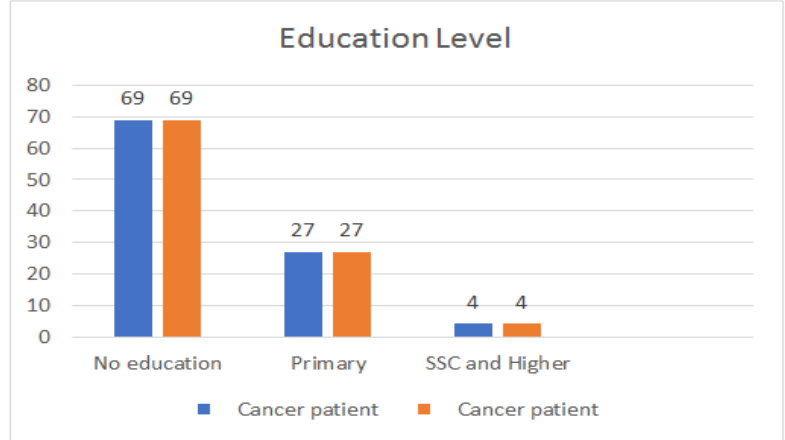

Fig-2: Educational level of the cervical cancer patients

In this study, $69 \%$ of cancer patients are noneducated. $27 \%$ are above primary level and only $4 \%$ are above the SSC level. 


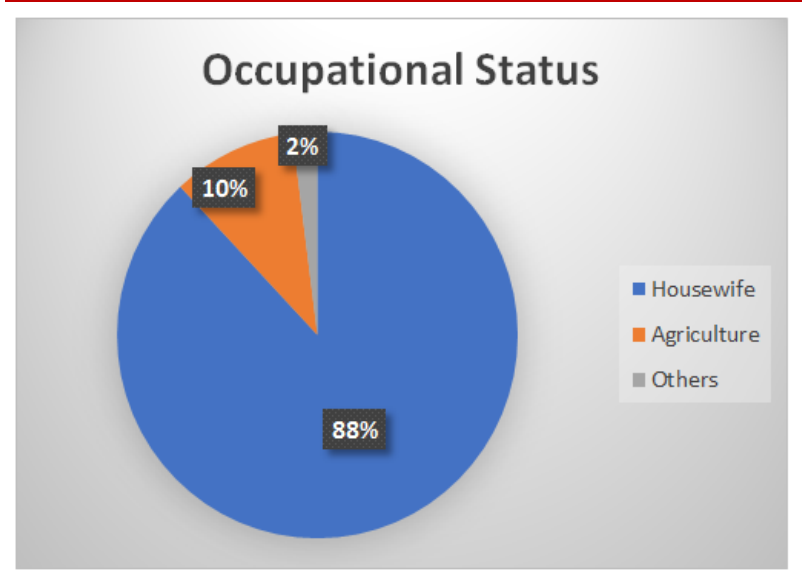

Fig-3: Occupational status of cervical cancer patients

In the present study, most (88\%) of the cancer patients are housewives. $2 \%$ are involved in agriculture and $10 \%$ are in other occupations.

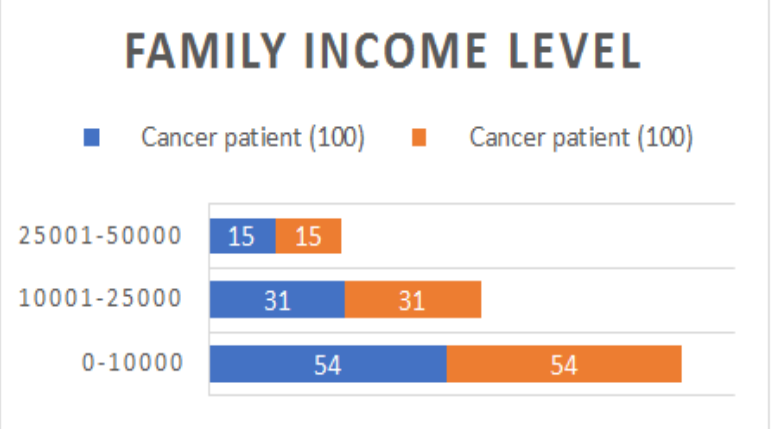

\section{Fig-4: Family income level of cervical cancer patients}

In this study monthly income level of fewer than 10000 takas was found in 54\% cases, less than 25000 takas in $31 \%$ cases, and in only $15 \%$ cases the income level was above the 25000 takas.

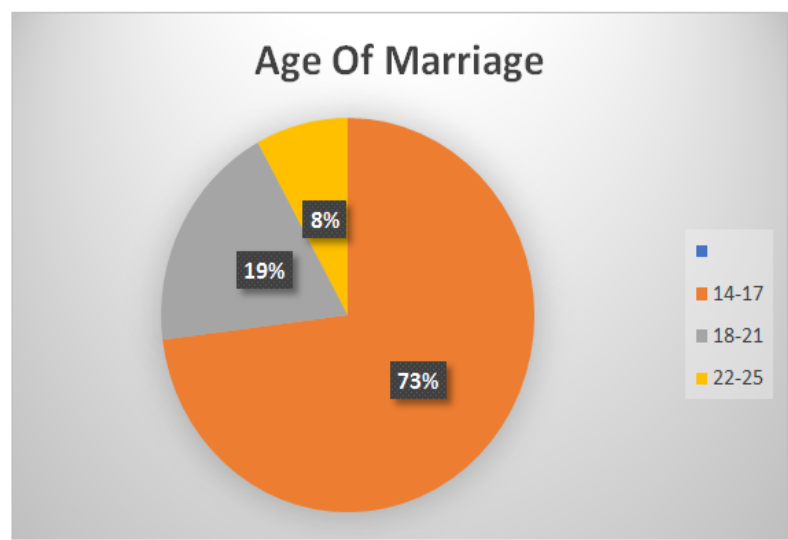

Fig-5: Age of Marriage of cervical cancer patient

The study shows that $73 \%$ of cancer patients were married in the early age group of 14-17 years, $19 \%$ in the $18-21$ years, and $8 \%$ in the $22-25$ years group.

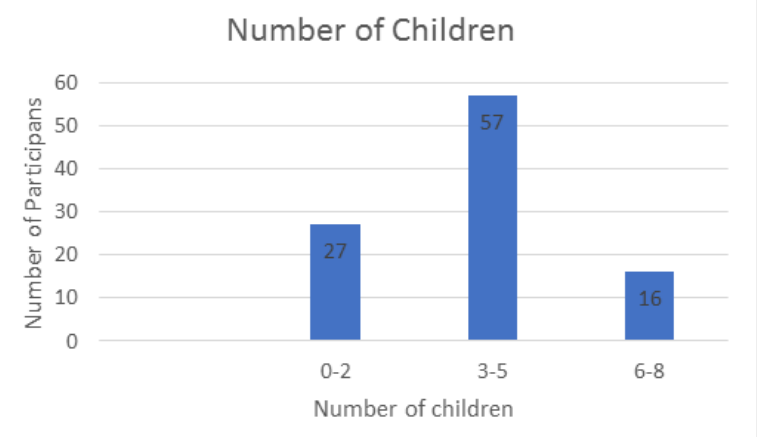

Fig-6: No of children of the cervical can patients

In the present study group of patients, $57 \%$ of them have $3-5$ children $27 \%$ of patients have $0-2$ children and $16 \%$ of them have $6-8$ children.

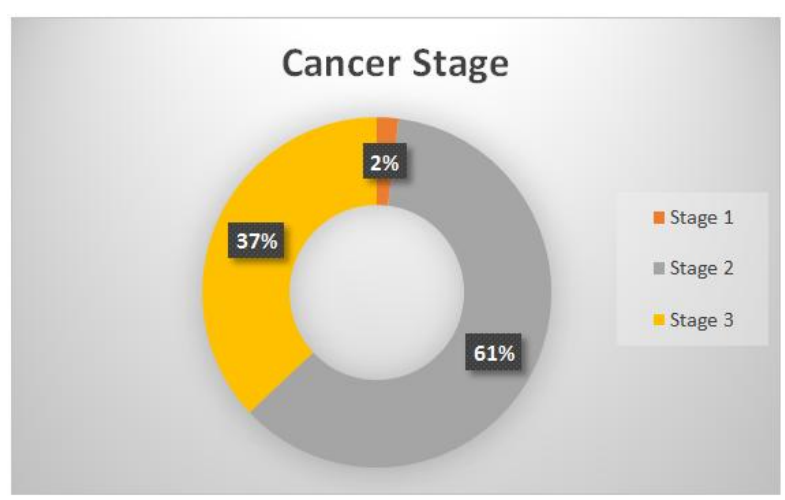

Fig-7: Cancer stage at admission of the cervical cancer patient

The present study shows that $98 \%$ of patients come at advanced stages (stage II \& III). Among them, $61 \%$ are in stage II and $37 \%$ are in stage III. Only $2 \%$ are presented in stage I disease.

\section{DISCUSSION}

Bangladesh is one of the most densely populated countries in the world. Ever since poverty has started to decline in the early 2000s, many economic development and lifestyle changes have occurred. The occurrence of cancer is one of the bads that came with the goods. Cancer is one of the NCD and is under observation to help reduce the mortality rate by NCD by $25 \%$ by 2025 . Globally, cervical cancer amounts to almost $12 \%$ of all female cancer. It is the fourth most common female cancer worldwide. An increased incidence of Cervical Cancer was observed with increasing age and parity and early and prolonged sexual period [3]. As cervical cancer grows slowly from precancerous lesions to advanced stages of cancer, it generally peaks in women in their 50s and 60s [4]. In this study, more than half of the patients were from the age group of 41-50 years and 20\% of the patients were of 30-40 years of age. Which coincides with various other studies. Educational level was also an important indicator of socio-economic status and its association 
Monirul Hassan \& Taslima Nigar; Sch Int J Obstet Gynec, Mar. 2021; 4(3): 58-62

with occupation, income level, and other lifestyle factors. In this study, almost $70 \%$ of the patients had no education. 27 had primary levels of education and only 4 had received an education of SSC and higher. Although not to a shocking degree similar to ours, the incidence rates of cervical cancer strikingly declined with increasing levels of education in another study conducted in India [5], where $56.5 \%$ of cancer study patients were found illiterate. Another study highlighted the importance of proper education of women of low socio-economic class to create awareness regarding hazards and risk factors of cervical cancer.[3] This study, in conjunction with other studies done at other similar demographics, showed that lower education level leads to unawareness regarding the disease and results in higher incident cases [3,6]. One of the factors that play a role in ill health is unemployment. For most individuals, work is one of the factors that keep meaning for their lives and gives an opportunity to participate in society. Studies have concluded that work allows a person to be a part of the social structure and makes them content in social, familial, and personal aspects of life. Without a job, the emotional, and oftentimes, physical wellbeing is jeopardized. Unemployment often leads to poverty, poor genital hygiene, low socioeconomic profile, lack of access to health services, and lack of awareness about healthcare, which contributes to a high prevalence in rural areas $[6$, 7]. In this study, the majority of the sample size were housewives $(\mathrm{n}=88)$, which showed a shocking similarity to another study conducted in Bangladesh.[8] of our study, $2 \%$ were involved in agriculture and 10 were involved in other occupations. This shows a link between occupational status and cancer incidence rates. Another socio-economic factor that played a role in cancer awareness and incidence cases was found to be the income level. Income level was studied differently from the occupational status of the study subjects, as income from other members of the family was also considered at this stage. Although the association made between socio-economic status and cervical cancer is not a direct association for the risk of cervical cancer, it is often referred to as a "disease of the poor, uneducated and underserved." This is because women with low family income, coupled with low education level and awareness are less likely to go for routine check-ups and screening, and also can hardly avail the best services available $[9,10]$. In the present study, over half the study population $(n=54)$ had a monthly income of 10,000 and less. 31 patients had a monthly family income of 10,001-25,000. Only 15 had a monthly income higher than 25,000. Most studies show the relationship between cervical cancer and age at first sexual intercourse, generally used as a surrogate measure of age at first HPV exposure. The mechanism that explains the risk of cervical carcinogenesis may be explained by the steroid hormonal influence on HPV infection that comes with early experience of first sexual intercourse and first pregnancy. The transformation zone of the cervical epithelium has been recognized as the site in which HPV infection tends to cause cancer, and the susceptibility of this area is believed to be related to its denudation of the stratified epithelium, thus facilitating exposure of the basal layer to HPV with minimal trauma [11]. Age at first marriage (AFM) is often used as a proxy measure for AFSI. An increased risk of infection of cervical carcinogenesis was consistently observed with decreasing age of first sexual intercourse (AFSI) [12]. The current study shows that almost $3 / 4^{\text {th }}(n=73)$ of cancer patients were married between the age of 14-17 years. Of the remaining, $19 \%$ were married between the age of 18-21 years and $8 \%$ at the age group of 22-25 years. In many case-control studies, high parity has constantly been associated with cervical cancer [13, 14]. Hormonal, traumatic, and immunological hypotheses have been reasoned as biologically plausible mechanisms to explain the association between parity and cervical neoplasia. In the present study, over half the study subjects had 3-5 kids, and 27 patients had less than 3 kids. The remaining 16 had more than 5 kids. This supports the findings from previous studies. The effect of our socio-economic status is also visible in the cancer stages of patients at the time of admission. The collected data shows only 2 patients were diagnosed with stage-1 $\mathrm{CC}$ at the time of admittance. The remaining 98patients were diagnosed with advanced stages of cancer, as 61 were diagnosed with stage- 2 CC and 37 were diagnosed with stage-3. This can be attributed to the lack of awareness and lack of proper and easily accessible screening methods, as cervical cancer can be determined in its early stages with proper screening [15-17]. Oftentimes incomplete knowledge about the disease can lead to improper diagnosis at the hands of inexperienced practitioners, which increases the likelihood of mistreatment of the disease and increases the risk of advanced stages of cancer [18, 19].

\section{LIMITATIONS OF THE STUDY}

The study was conducted with small sample size and in a single institute. Lack of awareness regarding the study topic also created some complications. Social and family pressure also discouraged many from participating in this study.

\section{CONCLUSION}

The socio-economical co-factors of cervical cancer were identified with this study. Some of these factors can be easily addressed. Correlation between these factors and increased incidence case of CC were noticed in this study. Regular cervical screenings are necessary to reduce the incidence and mortality from cervical cancer.

\section{RECOMMENDATION}

The study needs to be conducted with a larger sample size and in multiple institutes to receive data from a wider range of demographics. Knowledge about cervical cancer needs to be better shared between the 
Monirul Hassan \& Taslima Nigar; Sch Int J Obstet Gynec, Mar. 2021; 4(3): 58-62

general public. Proper treatment methods should be made common knowledge, and proper steps need to be taken to better address the socio-economic factors.

Conflict of Interest: None Declared.

\section{REFERENCES}

1. Fitzmaurice C, Dicker D, Pain A, Hamavid H, Moradi-Lakeh M, MacIntyre MF, Allen C, Hansen G, Woodbrook R, Wolfe C, Hamadeh RR. The global burden of cancer 2013. JAMA oncology. 2015 Jul 1;1(4):505-27.

2. Global Burden of Disease Cancer Collaboration. The global burden of cancer 2013. JAMA oncology. 2015 Jul 1;1(4):505.

3. Rajarao P, Hemanth Kumar B. Study of socio demographic profile of cancer cervix patients in tertiary care hospital, Karimnagar (Andhra Pradesh). Int J Biol Med Res. 2012;3(4):2306-10.

4. Chauhan R, Trivedi V, Rani R, Singh U. A hospital based study of clinical profile of cervical cancer patients of Bihar, an eastern state of India. of. 2016;4:2.

5. Bhattacharyya SK, Basu S, Banerjee S, Dastidar AG, Bagchi SR. An epidemiological survey of carcinoma cervix in north Bengal zone. Journal of the Indian Medical Association. 2000 Feb 1;98(2):60-1.

6. Kaverappa VB, Prakash B, Kulkarni P, Renuka M. Sociodemographic profile of patients with cervical cancer in a tertiary-care cancer hospital in Mysuru, Karnataka. International Journal of Medical Science and Public Health. 2015 Sep 1;4(9):118791.

7. Yumkhaibam SD, Ramalingam HS, Singh IY, Singh TT, Singh LJ, et al. (2016) Clinicoepidemiological Study of Cervical Carcinoma in Northeast India 6: 64-66.

8. Papri FS, Khanam Z, Islam F, Hakim MM. Knowledge and Awareness about risk factors of cervical cancer, its screening and vaccination among the women attending Chittagong Medical College Hospital. Chattagram Maa-O-Shishu Hospital Medical College Journal. 2015 Nov 16;14(2):57-60.

9. Juneja A, Sehgal A, Mitra AB, Pandey A. A survey on risk factors associated with cervical cancer. Indian Journal of Cancer. 2003 Jan 1; 40(1):15-22.

10. Hammouda D, Munoz N, Herrero R, Arslan A,
Bouhadef A, Oublil M, Djedeat B, Fontaniere B, Snijders P, Meijer C, Franceschi S. Cervical carcinoma in Algiers, Algeria: human papillomavirus and lifestyle risk factors. International journal of cancer. 2005 Jan 20; 113(3):483-9.

11. Bosch FX, Lorincz A, Muñoz N, Meijer CJ, Shah KV. The causal relation between human papillomavirus and cervical cancer. Journal of clinical pathology. 2002 Apr 1; 55(4):244-65.

12. Brinton LA, Hamman RF, Huggins GR, Lehman HF, Levine RS, Mailin K, Fraumeni Jr JF. Sexual and reproductive risk factors for invasive squamous cell cervical cancer. Journal of the National Cancer Institute. 1987 Jul 1; 79(1):23-30.

13. Muñoz N, Franceschi S, Bosetti C, Moreno V, Herrero R, Smith JS, Shah KV, Meijer CJ, Bosch FX, International Agency for Research on Cancer (IARC) Multicentric Cervical Cancer Study Group. Role of parity and human papillomavirus in cervical cancer: the IARC multicentric case-control study. The Lancet. 2002 Mar 30; 359(9312):1093101.

14. Deacon JM, Evans CD, Yule R, Desai M, Binns W, Taylor C, Peto J. Sexual behaviour and smoking as determinants of cervical HPV infection and of CIN3 among those infected: a case-control study nested within the Manchester cohort. British journal of cancer. 2000 Dec; 83(11):1565-72.

15. Hicks ML, Yap OS, Mathews R, Parham G. Disparities in cervical cancer screening, treatment and outcomes. Ethnicity and Disease. 2006 Jan 1; 16(2):S3.

16. Brewer N, Pearce N, Jeffreys M, White P, EllisonLoschmann L. Demographic differences in stage at diagnosis and cervical cancer survival in New Zealand, 1994-2005. Journal of Women's Health. 2009 Jul 1; 18(7):955-63.

17. Berraho M, Obtel M, Bendahhou K, Zidouh A, Errihani H, Benider A, Nejjari C. Sociodemographic factors and delay in the diagnosis of cervical cancer in Morocco. Pan African Medical Journal. 2012; 12(1).

18. Ibrahim A, Rasch V, Pukkala E, Aro AR. Predictors of cervical cancer being at an advanced stage at diagnosis in Sudan. International journal of women's health. 2011; 3:385.

19. Mayor S. A quarter of patients with cancer see their GP several times before being referred. 